\title{
Hausdorff's theorem for posets that satisfy the finite antichain property
}

\author{
by
}

Uri A b r a ham (Be'er Sheva) and Robert Bon net (Chambéry)

\begin{abstract}
Hausdorff characterized the class of scattered linear orderings as the least family of linear orderings that includes the ordinals and is closed under ordinal summations and inversions. We formulate and prove a corresponding characterization of the class of scattered partial orderings that satisfy the finite antichain condition (FAC).

Consider the least class of partial orderings containing the class of well-founded orderings that satisfy the FAC and is closed under the following operations: (1) inversion, (2) lexicographic sum, and (3) augmentation (where $\langle P, \preceq\rangle$ augments $\langle P, \leq\rangle$ iff $x \preceq y$ whenever $x \leq y$ ). We show that this closure consists of all scattered posets satisfying the finite antichain condition.

Our investigation also sheds some light on the natural (Hessenberg) sum of ordinals and the related product and exponentiation operations.
\end{abstract}

1. Introduction. The title of our paper refers to the well-known analysis of the scattered linear orderings made by Hausdorff [5]: Let $\mathcal{H}^{\text {linear }}$ be the closure of the class of well-ordered sets under inversions and wellordered summations. Then $\mathcal{H}^{\text {linear }}$ consists exactly of all linear scattered orderings (all definitions and needed preliminaries will be given below). We relax the requirement on linearity and obtain a corresponding result concerning the class of partially ordered sets that satisfy the finite antichain condition (FAC - every set of pairwise incomparable points is finite). Precisely, we shall prove the following theorem in Section 3.

Define $\mathcal{H}$ as the closure of the class of all FAC well-founded posets under lexicographic sums, inverses, and augmentations of the orderings. Then $\mathcal{H}$ is exactly the class of all scattered FAC posets.

1991 Mathematics Subject Classification: Primary 06B30, 54E45, 54E12; Secondary 06B05.

Key words and phrases: ordinals, partial orderings, scattered partial orderings, Hausdorff's theorem. 
In this formulation, the theorem is not an extension of Hausdorff's theorem because it (necessarily) allows augmentations, but we provide a finer investigation of the ranks of the posets involved and then obtain the theorem of Hausdorff as a particular case.

The proof is very elementary and should be accessible to readers with only a basic background in set theory. We therefore give all necessary definitions and some known results in the sequel of this introduction.

On posets. We recall here some definitions of elementary notions: posets, scattered posets, embedding, augmentation, etc. We recall some elementary facts. For example, an augmentation of a well-quasi-order (wqo) is wqo, and an augmentation of a scattered wqo is scattered.

If $P$ is a partially ordered set (poset) then $P$, or more formally $|P|$, denotes the universe of the ordering, and $\leq^{P}$ (or $\leq$ when no confusion can arise) denotes the ordering itself. We write $a<b$ for $a \leq b$ and $a \neq b$.

We say that $a$ and $b$ are incomparable $(a \perp b)$ when $a \not \leq b$ and $b \not \leq a$. An antichain is a set of pairwise incomparable elements. The Finite Antichain Condition (FAC) for a poset $P$ is the property that any antichain in $P$ is finite.

If $P$ is a poset, then $P^{*}$ denotes it inverse ordering, that is, $|P|=\left|P^{*}\right|$ and for every $p$ and $q, p \leq^{P^{*}} q$ iff $p \geq^{P} q$.

For any poset $P$ and $x \in P$ we define

$$
(\leq x)=\{y \in P \mid y \leq x\}, \quad(\perp x)=\{y \in P \mid y \perp x\} .
$$

$(<x),(\geq x)$, and $(>x)$ are defined similarly. We may write $(\leq x)^{P},(<x)^{P}$ etc. for emphasis.

1. We say that $P$ is a subposet of $Q$ if the universe of $P$ is a subset of the universe of $Q$ and whenever $p_{1}<^{P} p_{2}$ then $p_{1}<^{Q} p_{2}$.

2. If $P$ is a subposet of $Q$ such that for any $p_{1}, p_{2} \in|P|$ we have $p_{1}<^{P} p_{2}$ iff $p_{1}<^{Q} p_{2}$, then $P$ is said to be the restriction of $Q$ to $|P|$, and we write $P=Q \| P \mid$.

3. If $P$ is a subposet of $Q$ but they both have the same universe, then we say that $Q$ augments $P$. We denote by $\operatorname{aug}(P)$ the set of all possible augmentations of $P$. Clearly, every augmentation of a FAC poset satisfies the FAC as well.

4. A poset is well-founded if it has no infinite decreasing sequence. $P$ is well-founded iff there is a rank function for $P$, that is, an ordinal-valued function $r: P \rightarrow$ Ord such that $r(p)=\sup \left\{r(x)+1 \mid x<{ }^{P} p\right\}$ for every $p$ in $P$.

5. A transitive and reflexive relation is called a quasi-ordering. If every antichain is finite and every decreasing sequence is finite then the quasiordering is said to be a well-quasi-ordering. This is a very familiar term 
and a useful notion, but since we prefer to use partially ordered sets (rather than quasi-orderings) we will not use it, and hence our rather long (but self-explanatory) $F A C$ well-founded poset terminology.

6. Let $P$ and $Q$ be two posets; an embedding of $P$ into $Q$ is a one-to-one function $f$ from the universe of $P$ into the universe of $Q$ such that for every $a, b \in P, a<^{P} b$ iff $f(a)<^{Q} f(b)$. We say that $P$ is scattered if there is no embedding of $\mathbb{Q}$ (the chain of rational numbers) into $P$.

7. Given two posets $P$ and $Q$ we define the sum $P+Q$ as the poset obtained by putting every member of $P$ below every member of (a disjoint copy of) $Q$. The perpendicular sum $P \perp Q$ is defined by taking disjoint copies of $P$ and of $Q$ and making members of $P$ incomparable to members of $Q$. The Cartesian product $P \times Q$ is the partial ordering obtained by defining $(a, b) \leq\left(a^{\prime}, b^{\prime}\right)$ iff $a \leq^{P} a^{\prime}$ and $b \leq^{Q} b^{\prime}$.

8. The lexicographic ordering $P \cdot Q$ is obtained, roughly speaking, by replacing each point in $Q$ with a copy of $P$. This operation corresponds, more generally, to that of lexicographic sum $\sum_{q \in Q} P_{q}$ obtained by replacing each point $q$ in $Q$ with a copy $P_{q}$ of $P$. More formally, this lexicographic sum is the set $\bigcup_{q \in|Q|}|P| \times\{q\}$ endowed with the ordering $\langle p, q\rangle \leq\left\langle p^{\prime}, q^{\prime}\right\rangle$ if and only if $q<^{Q} q^{\prime}$, or $q=q^{\prime}$ and $p \leq^{P_{q}} p^{\prime}$.

9. If $X$ is a set of ordinals, then $\operatorname{Sup}(X)$ denotes the least ordinal that is strictly greater than all ordinals in $X$, while $\sup (X)$ is the least ordinal that is $\geq$ than all ordinals in $X$. That is, $\operatorname{Sup}(X)=\sup \{\alpha+1 \mid \alpha \in X\}$.

An initial segment $I$ of a poset $P$ is a subset of $|P|$ such that for every $p \in I,(\leq p) \subseteq I$. We denote by $\mathcal{I}(P)$ the set of all initial segments of $P$.

Lemma 1.1. Let $P$ be a poset. Then the following are equivalent:

1. $P$ is a FAC well-founded poset.

2. For every infinite sequence $\left\langle p_{i} \mid i \in \omega\right\rangle$ of points from $P$ there are $i<j$ with $p_{i} \leq{ }^{P} p_{j}$.

3. Any augmentation of $P$ is well-founded.

4. The ordered set $\langle\mathcal{I}(P), \subseteq\rangle$ is well-founded.

We will not prove the lemma (see Chapter 4 of Fraïssé [4]) but only comment that Ramsey's theorem can be used.

Lemma 1.2 (Bonnet and Pouzet 1969). The following are equivalent for any poset $P$.

1. $P$ is scattered and satisfies the FAC.

2. Every augmentation of $P$ is scattered.

Pr o o f. (See [2] and Fraïssé [4, Chapter 7].) We prove only one direction, namely that 1 implies 2 . Suppose that $P=\langle|P|,<\rangle$ is scattered and satisfies 
FAC, but $P^{\prime}=\left\langle|P|,<^{\prime}\right\rangle$ is a non-scattered augmentation of $P$. We first prove the following claim:

Suppose that $S \subseteq|P|$ is such that the restriction $P^{\prime}\lceil S$ is isomorphic to $\mathbb{Q}$. Then there is $q \in S$ such that the restriction of $P^{\prime}$ to $(\perp q)^{P} \cap S$ contains a copy of $\mathbb{Q}$.

Indeed, as $P$ is scattered and $S$ is not an infinite antichain, there are $a<^{P} b$ in $S$ that are consecutive elements of $S$, i.e. there is no $c \in S$ satisfying $a<^{P} c<^{P} b$. Further, $a<^{\prime} b$ and the open interval $(a, b)^{<^{\prime}}:=$ $\left\{c \in|S| \mid a<^{\prime} c<^{\prime} b\right\}$ is order-isomorphic to $\mathbb{Q}$. Also, for $c \in(a, b)^{<^{\prime}}$, we have $c \perp a$ or $c \perp b$ in $P$. Let $D_{a}=\left\{c \in(a, b)^{<^{\prime}}: c \perp a\right\}$ and $D_{b}=\left\{c \in(a, b)^{<^{\prime}}: c \perp b\right\}$. Then $D_{a} \cup D_{b}$ is a partition of $(a, b)^{<^{\prime}}$ into two sets and hence one of the sets is not scattered in $<^{\prime}$ and contains a copy of $\mathbb{Q}$. This proves the claim.

By repeatedly applying the claim, one obviously gets an infinite antichain of $P$, which is impossible.

Note that the class of FAC scattered posets contains the class of FAC well-founded posets and is closed under lexicographic sums, inverses, and augmentations of the orderings (that follows from 1.2).

On ordinals. We recall some elementary facts concerning ordinal operations that we shall use and then proceed to investigate the Hessenberg based operations on the ordinals (which we believe to be new, despite their simplicity). Several textbooks in set theory can be consulted; for example Sierpiński [8], Fraïssé [4], or Lévy [7].

The Cantor normal form of an ordinal $\alpha$,

$$
\alpha=\omega^{\alpha(0)} m(0)+\ldots+\omega^{\alpha(i)} m(i)+\ldots+\omega^{\alpha(l)} m(l),
$$

is determined by a non-empty decreasing sequence $\alpha(0)>\alpha(1)>\ldots>$ $\alpha(l) \geq 0$ of ordinals, and a sequence of natural numbers $m(i) \geq 0$. (Except for $\alpha=0$, it is possible to demand that each $m(i)>0$, but there are occasions where it seems to be convenient to allow for $m(i)=0$.)

Each $\omega^{\alpha(i)}$ with $m(i)>0$ is called here a block of $\alpha$, and the least block is called the tip of $\alpha$. So, for example, if $\alpha=\omega^{\omega} 2+\omega^{7}+\omega^{3} 5$, then $\operatorname{tip}(\alpha)=\omega^{3}$, and if $\alpha=\omega+5$ then $\operatorname{tip}(\alpha)=1$.

The ordering of two ordinals can be determined by their coefficient series: Suppose that $\alpha_{1}$ and $\alpha_{2}$ are two ordinals. By allowing $m(i)=0$ we can assume that they have the same sequence of exponents

$$
\alpha(0)>\ldots>\alpha(l) \geq 0 .
$$

Let $m_{1}(i)$ and $m_{2}(i)$ for $i \leq l$ be the respective coefficients of $\alpha_{1}$ and $\alpha_{2}$. 
Then $\alpha_{1}<\alpha_{2}$ iff

$$
\left\langle m_{1}(0), \ldots, m_{1}(l)\right\rangle \prec\left\langle m_{2}(0), \ldots, m_{2}(l)\right\rangle
$$

where $\prec$ is the lexicographic ordering of sequences of natural numbers.

Definition. The natural sum (or Hessenberg sum) of two ordinals is defined by adding the coefficients of their normal forms as though these were polynomials in $\omega$. For example, if $\alpha=\omega^{\omega+\omega} 8+\omega^{7} 3$ and $\beta=\omega^{\omega}+\omega^{7}+$ $\omega^{2}+\omega^{0} 4$ then $\alpha \oplus \beta=\omega^{\omega+\omega} 8+\omega^{\omega}+\omega^{7} 4+\omega^{2}+4$.

Carruth [3] characterized $\alpha \oplus \beta$ as the maximal order type of $A \cup B$, where $A$ and $B$ are sets of ordinals of order type $\alpha$ and $\beta$. We indicate a proof:

Relying on the criterion given above for the ordering of ordinals, one can see that the two-place function $\alpha \oplus \beta$ is strictly increasing in both arguments. This can be used to prove that if $A, B$ are sets of ordinals of order type $\alpha$ and $\beta$ respectively, then $\operatorname{tp}(A \cup B) \leq \alpha \oplus \beta$ (here $\operatorname{tp}(X)$ is the order type of $X$ ). Indeed, define a function $f$ by setting

$$
f(\gamma)=\operatorname{tp}(A \cap \gamma) \oplus \operatorname{tp}(B \cap \gamma)
$$

for every $\gamma \in A \cup B$. Then $f: A \cup B \rightarrow \alpha \oplus \beta$ is order preserving, and hence $\operatorname{tp}(A \cup B) \leq \alpha \oplus \beta$.

On the other hand, $\alpha \oplus \beta$ (as a set of ordinals) is clearly the order type of the union $A \cup B$ of two disjoint sets of ordinals with $\operatorname{tp}(A)=\alpha$ and $\operatorname{tp}(B)=\beta$. This proves Carruth's characterization.

The natural presentation of $\alpha \oplus \beta$ as the union $A \cup B$ as above has the following property: If $\gamma<\alpha \oplus \beta$ then $\gamma=\operatorname{tp}(A \cap \gamma) \oplus \operatorname{tp}(B \cap \gamma)$. So

$$
\text { If } \gamma<\alpha \oplus \beta \text {, then } \gamma=\alpha_{0} \oplus \beta_{0} \text { where } \alpha_{0} \leq \alpha \text { and } \beta_{0} \leq \beta
$$

(and $\alpha_{0}<\alpha$ or $\beta_{0}<\beta$ ). (Do not be tempted to think that if $\alpha<\gamma<\alpha \oplus \beta$ then $\gamma=\alpha \oplus \beta_{0}$ for some $\beta_{0}<\beta$.)

The following remarks are for completeness, since we shall not use them in this paper. As Fraïssé [4, Chapter 7.2] remarks, for any two ordinals $\alpha$ and $\beta$ we have

$$
\sigma=\operatorname{Sup}_{\alpha^{\prime}<\alpha, \beta^{\prime}<\beta} \alpha^{\prime} \oplus \beta^{\prime}<\alpha \oplus \beta .
$$

The following, however, is easy to prove:

$$
\text { If } \alpha \text { is limit and } \operatorname{tip}(\alpha) \leq \operatorname{tip}(\beta) \text { then } \alpha \oplus \beta=\operatorname{Sup}_{\alpha^{\prime}<\alpha} \alpha^{\prime} \oplus \beta \text {. }
$$

It follows from this that, for limit ordinals $\alpha$ and $\beta$,

$$
\alpha \oplus \beta=\max \left\{\operatorname{Sup}_{\alpha^{\prime}<\alpha} \alpha^{\prime} \oplus \beta, \operatorname{Sup}_{\beta^{\prime}<\beta} \alpha \oplus \beta^{\prime}\right\} .
$$


Definition. The natural sum operation is used to define a product operation $\alpha \odot \beta$ on the ordinals by the following relations:

$$
\begin{aligned}
\alpha \odot 0 & =0, \\
\alpha \odot(\beta+1) & =(\alpha \odot \beta) \oplus \alpha, \\
\alpha \odot \beta & =\operatorname{Sup}\{\alpha \odot \gamma \mid \gamma<\beta\} \quad \text { for limit } \beta .
\end{aligned}
$$

We say that $\alpha \odot \beta$ is the Hessenberg based product of $\alpha$ and $\beta$. As usual, parentheses may be deleted. For instance, $\alpha \oplus(\beta \odot \gamma) \oplus \delta$ is denoted by $\alpha \oplus \beta \odot \gamma \oplus \delta$.

The operation $\odot$ is not the same as the Hessenberg operation $\alpha \otimes \beta$ which is obtained from the normal forms of $\alpha$ and $\beta$ viewed as polynomials and multiplied accordingly. In particular, $\alpha \otimes \beta$ is commutative, but $\odot$ is not. For example,

$$
\begin{gathered}
\omega \otimes 2=2 \otimes \omega=\omega+\omega, \\
\omega \odot 2=\omega \oplus \omega=\omega+\omega, \quad 2 \odot \omega=\operatorname{Sup}\{2 \odot n \mid n<\omega\}=\omega .
\end{gathered}
$$

The function $\alpha \odot \beta$ is strictly increasing in the right variable, continuous in the right variable, and non-decreasing in the left variable. $\alpha \otimes \beta$ is strictly increasing in both coordinates, but it is not continuous. We always have (prove by induction on $\beta$ )

$$
\alpha \beta \leq \alpha \odot \beta \leq \alpha \otimes \beta,
$$

where $\alpha \beta$ is the usual ordinal multiplication. Strict inequalities are possible; for instance, with $\alpha=\omega+1$ and $\beta=\omega+2$, we have: $\alpha \beta=\omega^{2}+\omega 2+1$, $\alpha \odot \alpha=\omega^{2}+\omega+1, \alpha \odot \beta=\omega^{2}+\omega 2+2$, and $\alpha \otimes \beta=\omega^{2}+\omega 3+2$.

We now recall a well-known formula for the product of ordinals, and show that a similar result also holds for the Hessenberg based product. For this we decompose any ordinal $\beta$ into its infinite parts and the finite (possibly empty) tip.

LEMma 1.3. If $\beta=\beta_{0}+k$ where $\beta_{0}$ is limit or 0 and $k \in \omega$, then for any $\alpha$ with Cantor normal form $\alpha=\omega^{\alpha(0)} m(0)+\ldots+\omega^{\alpha(l)} m(l)$ (where $m(0)>0)$,

$$
\begin{gathered}
\alpha \beta=\omega^{\alpha(0)} \beta_{0}+\alpha k=\omega^{\alpha(0)} \beta_{0} \oplus \alpha k, \\
\alpha \odot \beta=\omega^{\alpha(0)} \beta_{0} \oplus \alpha \odot k .
\end{gathered}
$$

Hence if $\beta=\beta_{0}+0$ is a limit ordinal then $\alpha \beta=\alpha \odot \beta=\omega^{\alpha(0)} \beta$.

Proof. We only prove (3), but the proof for (2) is the same. The proof is by induction on $\beta$. If $\beta=0$ then $\beta_{0}=0, k=0$ and (3) is trivial. If $\beta=\beta^{\prime}+1$ is a successor ordinal, and $\beta=\beta_{0}+k+1$ where $\beta_{0}$ is the last limit ordinal (or zero) below $\beta$, then $\beta^{\prime}=\beta_{0}+k$ and, by the inductive assumption,

$$
\alpha \odot \beta=\alpha \odot \beta^{\prime} \oplus \alpha=\omega^{\alpha(0)} \beta_{0} \oplus \alpha \odot k \oplus \alpha=\omega^{\alpha(0)} \beta_{0} \oplus \alpha \odot(k+1) .
$$


Now if $\beta$ is limit, then $\beta=\beta_{0}, k=0$ and we shall prove that $\alpha \odot \beta=\omega^{\alpha(0)} \beta$. By definition

$$
\alpha \odot \beta=\operatorname{Sup}_{\beta^{\prime}<\beta} \alpha \odot \beta^{\prime} .
$$

There are two possibilities.

1. $\beta$ is a limit of limit ordinals $\beta_{i}, i \in I$. Then

$$
\alpha \odot \beta=\operatorname{Sup}_{i \in I} \alpha \odot \beta_{i}=\operatorname{Sup}_{i \in I} \omega^{\alpha(0)} \beta_{i}=\omega^{\alpha(0)} \beta
$$

because (regular) ordinal multiplication is continuous on the right.

2. $\beta$ has the form $\beta=\gamma+\omega$ where $\gamma$ is limit or 0 . Then

$$
\alpha \odot \beta=\operatorname{Sup}_{n \in \omega} \alpha \odot(\gamma+n)=\operatorname{Sup}_{n \in \omega} \omega^{\alpha(0)} \gamma \oplus \alpha \odot n .
$$

Observe first that $\operatorname{Sup}_{n \in \omega} \alpha \odot n=\omega^{\alpha(0)} \omega$. So if $\beta=\omega$ then $\gamma=0$,

$$
\alpha \odot \beta=\operatorname{Sup}_{n \in \omega} \alpha \odot n=\omega^{\alpha(0)} \beta
$$

and we are done. Thus we assume $\gamma \geq \omega$. But $\omega^{\alpha(0)} \gamma \oplus \alpha \odot n=\omega^{\alpha(0)} \gamma+\alpha \odot n$, because $\operatorname{tip}\left(\omega^{\alpha(0)} \gamma\right) \geq \omega^{\alpha(0)} \omega>\alpha \odot n$. So, by the right-continuity property of ordinal addition, (4) gives

$$
\alpha \odot \beta=\omega^{\alpha(0)} \gamma+\omega^{\alpha(0)} \omega=\omega^{\alpha(0)}(\gamma+\omega)=\omega^{\alpha(0)} \beta .
$$

Corollary 1.4. Distributivity holds on the right:

$$
\alpha \odot\left(\beta_{1} \oplus \beta_{2}\right)=\alpha \odot \beta_{1} \oplus \alpha \odot \beta_{2} .
$$

Proof. This can be proved by induction on $\gamma=\beta_{1} \oplus \beta_{2}$. The case $\gamma=0$ is trivial, and the case of $\gamma$ a successor is easy. So assume that $\gamma$ is a limit ordinal, that is, both $\beta_{1}$ and $\beta_{2}$ are limit ordinals. Then

$$
\alpha \odot \gamma=\omega^{\alpha(0)} \gamma=\omega^{\alpha(0)}\left(\beta_{1} \oplus \beta_{2}\right)=\omega^{\alpha(0)} \beta_{1} \oplus \omega^{\alpha(0)} \beta_{2}=\alpha \odot \beta_{1} \oplus \alpha \odot \beta_{2} .
$$

We have used the formula $\omega^{\delta}\left(\beta_{1} \oplus \beta_{2}\right)=\omega^{\delta} \beta_{1} \oplus \omega^{\delta} \beta_{2}$, which can be easily computed.

Corollary 1.5. $\odot$ is associative: $(\alpha \odot \beta) \odot \gamma=\alpha \odot(\beta \odot \gamma)$.

Proof. We proceed by induction on $\gamma$. The successor case is handled as follows:

$$
\begin{aligned}
& (\alpha \odot \beta) \odot(\gamma+1)=(\alpha \odot \beta) \odot \gamma \oplus \alpha \odot \beta, \\
& \alpha \odot(\beta \odot(\gamma+1))=\alpha \odot(\beta \odot \gamma \oplus \beta)=\alpha \odot(\beta \odot \gamma) \oplus \alpha \odot \beta .
\end{aligned}
$$

So we see that the two terms are equal by the induction hypothesis. (To get the second equality, we have used distributivity.) 
The case where $\gamma$ is a limit ordinal is as follows:

$$
\begin{aligned}
(\alpha \odot \beta) \odot \gamma & =\operatorname{Sup}_{\gamma^{\prime}<\gamma}(\alpha \odot \beta) \odot \gamma^{\prime} \\
& =\operatorname{Sup}_{\gamma^{\prime}<\gamma} \alpha \odot\left(\beta \odot \gamma^{\prime}\right)=\alpha \odot(\beta \odot \gamma) .
\end{aligned}
$$

We have used here the fact that $\operatorname{Sup}_{\gamma^{\prime}<\gamma}\left(\beta \odot \gamma^{\prime}\right)=\beta \odot \gamma$ by definition, and the continuity property on the right, which says that if $\operatorname{Sup}_{g \in G} \beta_{g}=\beta$ is a limit ordinal, then $\operatorname{Sup}_{g \in G} \alpha \odot \beta_{g}=\alpha \odot \beta$.

Definition. In the following step $\odot$ is used to define the Hessenberg based exponentiation, which we denote by the superscript $\mathrm{H}$ (as in $\alpha^{\mathrm{H} \beta}$ ). Whenever it is perfectly clear that we deal with the Hessenberg based exponentiation, we shall omit $\mathrm{H}$ :

$$
\begin{array}{rlrl}
\alpha^{0} & =1 \text { for } \alpha>0 & & \left(\text { but } 0^{\beta}=0\right), \\
\alpha^{\beta+1} & =\alpha^{\beta} \odot \alpha, & \\
\alpha^{\beta} & =\operatorname{Sup}\left\{\alpha^{\gamma} \mid \gamma<\beta\right\} & \text { for limit } \beta .
\end{array}
$$

Lemma 1.6. The usual rules hold for the Hessenberg based exponentiation:

$$
\begin{aligned}
\alpha^{\beta+\gamma} & =\alpha^{\beta} \odot \alpha^{\gamma}, \\
\left(\alpha^{\beta}\right)^{\gamma} & =\alpha^{\beta \gamma} .
\end{aligned}
$$

Proof. All exponentiations are Hessenberg based exponentiations. We first show (5). The proof is by induction on $\gamma$. The case $\gamma=0$ is trivial. Look at the following for $\gamma+1$. By the definition of exponentiation and by the inductive assumption we have $\alpha^{\beta+\gamma+1}=\alpha^{\beta+\gamma} \odot \alpha=\left(\alpha^{\beta} \odot \alpha^{\gamma}\right) \odot \alpha=$ $\alpha^{\beta} \odot\left(\alpha^{\gamma} \odot \alpha\right)=\alpha^{\beta} \odot \alpha^{\gamma+1}$.

Now, for $\gamma$ a limit ordinal, we argue as follows: $\alpha^{\beta+\gamma}=\operatorname{Sup}_{\gamma^{\prime}<\gamma} \alpha^{\beta+\gamma^{\prime}}=$ $\operatorname{Sup}_{\gamma^{\prime}<\gamma} \alpha^{\beta} \odot \alpha^{\gamma^{\prime}}=\alpha^{\beta} \odot \alpha^{\gamma}$. This last equality is derived from the definition of $\mu \odot \delta$ when $\delta$ is a limit ordinal, and from the fact that $\operatorname{Sup}_{\gamma^{\prime}<\gamma} \alpha^{\gamma^{\prime}}=\alpha^{\gamma}$. So (5) holds.

The case $\gamma=0$ for (6) is trivial. Look at the following for (6) in the successor case $\gamma+1$ :

$$
\left(\alpha^{\beta}\right)^{\gamma+1}=\left(\alpha^{\beta}\right)^{\gamma} \odot \alpha^{\beta}=\alpha^{\beta \gamma} \odot \alpha^{\beta}=\alpha^{\beta \gamma+\beta}=\alpha^{\beta(\gamma+1)} .
$$

Here, we have used the induction hypothesis in the second equality, and (5) in the third equality.

For $\gamma$ limit, (6) follows from

$$
\left(\alpha^{\beta}\right)^{\gamma}=\operatorname{Sup}_{\gamma^{\prime}<\gamma}\left(\alpha^{\beta}\right)^{\gamma^{\prime}}=\operatorname{Sup}_{\gamma^{\prime}<\gamma} \alpha^{\left(\beta \gamma^{\prime}\right)}=\alpha^{\beta \gamma} .
$$


For many ordinals $\mu$, we have $\mu^{\mathrm{H} \alpha}=\mu^{\alpha}$ for all $\alpha$ 's. That is, the Hessenberg based exponentiation coincides with the regular one. For example:

Lemma 1.7. For every $\alpha, \omega^{\mathrm{H} \alpha}=\omega^{\alpha}$.

P r o of. We proceed by induction on $\alpha$. The cases $\alpha=0,1$ and $\alpha$ a limit ordinal are very easy. Now consider the successor case:

$$
\omega^{\mathrm{H}(\alpha+1)}=\omega^{\mathrm{H} \alpha} \odot \omega=\omega^{\alpha} \odot \omega=\omega^{\alpha} \omega=\omega^{\alpha+1} .
$$

For instance, note that $(\omega+2)^{\omega+1}<(\omega+2)^{\mathrm{H}(\omega+1)}$.

The following lemma is used in the proof of Lemma 1.11.

Lemma 1.8. If $\gamma<\alpha \odot \beta$ then for some $k<\omega$,

$$
\gamma=\alpha_{1} \oplus \ldots \oplus \alpha_{k} \oplus \alpha \odot \beta_{0}
$$

where $\alpha_{i}<\alpha$ for every $i$ and $\beta_{0}<\beta$, and in fact $\beta_{0}+k \leq \beta$.

Moreover, if $\gamma^{\prime}<\alpha_{1} \oplus \ldots \oplus \alpha_{k} \oplus \alpha \odot \beta_{0}$, then for some $l \in \omega$,

$$
\gamma^{\prime}=\alpha_{1}^{\prime} \oplus \ldots \oplus \alpha_{k}^{\prime} \oplus \alpha_{k+1}^{\prime} \oplus \ldots \alpha_{k+l}^{\prime} \oplus \alpha \odot \beta_{0}^{\prime}
$$

where $\alpha_{i}^{\prime} \leq \alpha_{i}$ for each $1 \leq i \leq k$ and either

1. $l=0$ and $\beta_{0}^{\prime}=\beta_{0}$, or

2. $l>0$ and then $\beta_{0}^{\prime}+l \leq \beta_{0}$.

$\operatorname{Proof}$. We prove the first statement by induction on $\beta$. If $\beta$ is 0 or is a limit ordinal the statement is obvious. So assume that $\gamma<\alpha \odot(\beta+1)=$ $\alpha \odot \beta \oplus \alpha$. Then (by (1))

$$
\gamma=\mu \oplus \alpha_{0} \quad \text { where } \mu \leq \alpha \odot \beta \text { and } \alpha_{0} \leq \alpha .
$$

If $\alpha_{0}=\alpha$ then necessarily $\mu<\alpha \odot \beta$ and the inductive hypothesis applied to $\mu$ gives $\mu=\alpha_{1} \oplus \ldots \oplus \alpha_{k} \oplus \alpha \odot \beta_{0}$ with $\beta_{0}+k \leq \beta$. Then

$$
\gamma=\alpha_{1} \oplus \ldots \oplus \alpha_{k} \oplus \alpha \odot \beta_{0} \oplus \alpha=\alpha_{1} \oplus \ldots \oplus \alpha_{k} \oplus \alpha \odot\left(\beta_{0}+1\right),
$$

and $\beta_{0}+1+k \leq \beta+1$ as required.

If $\alpha_{0}<\alpha$, then there are two subcases: $\mu=\alpha \odot \beta$ and $\mu<\alpha \odot \beta$. The first subcase is obvious (giving $\gamma=\alpha_{0} \oplus \alpha \odot \beta$ ), and the inductive hypothesis is applied in the second subcase.

The "moreover" part of the lemma is a consequence of the first part. If

$$
\gamma^{\prime}<\left[\alpha_{1} \oplus \ldots \oplus \alpha_{k}\right] \oplus\left[\alpha \odot \beta_{0}\right]
$$

then apply (1) to the Hessenberg sum of the two ordinals enclosed in the square brackets, and write $\gamma^{\prime}=\gamma_{0} \oplus \gamma_{1}$ with $\gamma_{0} \leq \alpha_{1} \oplus \ldots \oplus \alpha_{k}$, and $\gamma_{1} \leq \alpha \odot \beta_{0}$. For $\gamma_{1}<\alpha \odot \beta_{0}$, use the first part of the lemma.

On ranks of posets. Any well-founded poset $A$ has an associated rank function $\mathrm{rk}: A \rightarrow$ Ord satisfying

$$
\operatorname{rk}(a)=\operatorname{Sup}\left\{\operatorname{rk}(x) \mid x<^{A} a\right\} .
$$


The range of rk, that is, $\operatorname{Sup}_{a \in A} \operatorname{rk}(a)$, is called the length of $A$ and is denoted by $\operatorname{rk}(A)$ for simplicity. If $\varrho: A \rightarrow$ Ord is any order preserving function, then $\operatorname{rk}(a) \leq \varrho(a)$ for every $a \in A$. Thus $\operatorname{rk}(A)$ is the least ordinal into which there is an order preserving function from $A$. The rank function "leaves no gaps": if $\gamma<\operatorname{rk}(a)$ then for some $a^{\prime}<{ }^{A} a$ we have $\gamma=\operatorname{rk}\left(a^{\prime}\right)$.

The basic reason that the natural sum is so useful for us is the following simple lemma.

LEmma 1.9. If $r_{X}: X \rightarrow \alpha$ and $r_{Y}: Y \rightarrow \beta$ are the rank functions of the well-founded posets $X$ and $Y$, then the rank function rk on $X \times Y$ satisfies

$$
\operatorname{rk}(\langle x, y\rangle)=r_{X}(x) \oplus r_{Y}(y) .
$$

Proof. Since $\oplus$ is order preserving in both coordinates, the expression $r_{X}(x) \oplus r_{Y}(y)$ on the right-hand side is an order preserving function from $X \times Y$ into $\alpha \oplus \beta$. From this follows the " $\leq$ " part of the equality. Equality follows from (1), namely if $\gamma<r_{X}(x) \oplus r_{Y}(y)$ then $\gamma=\alpha_{0} \oplus \beta_{0}$ for some $\alpha_{0} \leq r_{X}(x), \beta_{0} \leq r_{Y}(y)$. So if (7) is violated, then it is violated by some minimal pair $\langle x, y\rangle$ in $X \times Y$. But then $\operatorname{rk}(\langle x, y\rangle)=\gamma<r_{X}(x) \oplus r_{Y}(y)$ implies that $\gamma=\alpha_{0} \oplus \beta_{0}$ for some $\alpha_{0} \leq r_{X}(x), \beta_{0} \leq r_{Y}(y)$ and strict inequality must hold at least once. Say $\alpha_{0}<r_{X}(x)$, and then there is $x_{0}<x$ with $\alpha_{0}=\operatorname{rk}_{X}\left(x_{0}\right)$ and $y_{0} \leq y$ with $\operatorname{rk}_{Y}\left(y_{0}\right)=\beta_{0}$. Now, as $\left\langle x_{0}, y_{0}\right\rangle<\langle x, y\rangle$, we have $\operatorname{rk}\left(\left\langle x_{0}, y_{0}\right\rangle\right)=\operatorname{rk}_{X}\left(x_{0}\right) \oplus \operatorname{rk}_{Y}\left(y_{0}\right)=\gamma$ which is a contradiction! (Compare with Fraïssé [4], and see Abraham [1] for related results.)

On the antichain rank. Observe that $P$ satisfies the FAC iff the set of antichains of $P$ under inverse inclusion forms a well-founded poset. That is, there is no infinite strictly $\subset$-increasing sequence of antichains in $P$. When $P$ satisfies the FAC, we denote by $\langle\mathcal{A}(P), \supset\rangle$ the poset of all nonempty antichains of $P$ under inverse inclusion. The rank function on $\mathcal{A}(P)$ is denoted by $\mathrm{rk}_{\mathcal{A}}$, and its image (which is an ordinal) is called the length (or the rank) of $\mathcal{A}(P)$. For simplicity of notation we denote this rank by $\mathrm{rk}_{\mathcal{A}}(P)$. (This may be confusing if $P$ itself is a finite antichain.) Denote by $\mathcal{A}^{+}(P)=\mathcal{A}(P) \cup\{\emptyset\}$ the collection of all antichains of $P$, including the empty antichain, which is not in $\mathcal{A}(P)$, and extend $\mathrm{rk}_{\mathcal{A}}$ on the empty antichain by defining $\operatorname{rk}_{\mathcal{A}}(\emptyset)=\operatorname{rk}_{\mathcal{A}}(P)$.

We have the following results:

LEMMA 1.10. If $P$ and $Q$ are FAC posets with antichain rank functions $r_{P}$ and $r_{Q}$, then the perpendicular sum $R=P \perp Q$ is also a FAC poset and its rank function $\mathrm{rk}_{\mathcal{A}}$ satisfies

$$
\operatorname{rk}_{\mathcal{A}}(X)=r_{P}(X \cap P) \oplus r_{Q}(X \cap Q)
$$

for every antichain $X$ in $R$ (including the empty antichain). In particular, $\mathrm{rk}_{\mathcal{A}}(R)=r_{P}(P) \oplus r_{Q}(Q)$. 
Proof. The proof is a direct consequence of Lemma 1.9 and the fact that $\mathcal{A}^{+}(P \perp Q)=\mathcal{A}^{+}(P) \times \mathcal{A}^{+}(Q)$. (Recall that $\mathcal{A}^{+}(X)=\mathcal{A}(X) \cup\{\emptyset\}$ is the set of all antichains of $X$, including the empty one.) So $\operatorname{rk}_{\mathcal{A}}(R)=$ $\mathrm{rk}_{\mathcal{A}}(\emptyset)=r_{P}(\emptyset) \oplus r_{Q}(\emptyset)=r_{P}(P) \oplus r_{Q}(Q)$.

Lemma 1.11. If $P$ and $Q$ are $F A C$ posets, and $R=P \cdot Q$ is the lexicographic product (that is, the lexicographic sum of copies of $P$ along $Q$ ), if $r_{P}$ and $r_{Q}$ are the antichain rank functions on $\mathcal{A}(P)$ and $\mathcal{A}(Q)$, then $R$ is a FAC poset as well, and the length of $\mathcal{A}(R)$ is $r_{P}(P) \odot r_{Q}(Q)$.

Proof. Lemma 1.8 is used in the proof. Any antichain $X$ in $R$ can be represented as a union of $P$-antichains along some $Q$-antichain. That is, $X=\bigcup\left\{A_{q} \mid q \in B\right\}$ where $B$ is an antichain in $Q$ and each $A_{q}$ is an antichain in $P$. If $B=\{q(1), \ldots, q(n)\}$, and $\alpha=r_{P}(P)$, define

$$
f(X)=r_{P}\left(A_{q(1)}\right) \oplus r_{P}\left(A_{q(2)}\right) \oplus \ldots \oplus r_{P}\left(A_{q(n)}\right) \oplus \alpha \odot r_{Q}(B) .
$$

We shall prove that $f$ is the rank function for $R$. Having done so, we can put $X=\emptyset$ to obtain the lemma. First we prove the following:

Claim 1. The function $f$ is order reversing: If $X_{1} \subset X_{2}$ are two antichains in $R$, then $f\left(X_{1}\right)>f\left(X_{2}\right)$.

This claim implies that $r_{R}(X) \leq f(X)$ for any antichain $X$ in $R\left(r_{R}\right.$ is the antichain rank for $R$ ).

Proof of Claim 1. Suppose that $X_{1}$ is represented with $P$-antichains $A_{q}^{1}$ for $q \in B_{1}$, and $X_{2}$ is represented with $A_{q}^{2}$ for $q \in B_{2}$. Then $X_{1} \subset X_{2}$ implies that $B_{1} \subseteq B_{2}$ and for $q \in B_{1}$ and $A_{q}^{1} \subseteq A_{q}^{2}$. There are two cases to consider: when $B_{1}=B_{2}$ and when $B_{1} \subset B_{2}$.

In the first case it is easy to see that $f\left(X_{1}\right)>f\left(X_{2}\right)$, because the Hessenberg sum is (strictly) order preserving in all coordinates.

In the second case, let $l \geq 1$ be the number of points in $B_{2}$ not in $B_{1}$. Then $r_{Q}\left(B_{2}\right)+l \leq r_{Q}\left(B_{1}\right)$. This is because if $B_{2}=B_{1} \cup\left\{b_{1}, \ldots, b_{l}\right\}$, then

$$
\operatorname{rk}_{Q}\left(B_{1}\right)>\operatorname{rk}_{Q}\left(B_{1} \cup\left\{b_{1}\right\}\right)>\operatorname{rk}_{Q}\left(B_{1} \cup\left\{b_{1}, b_{2}\right\}\right)>\ldots>\operatorname{rk}_{Q}\left(B_{2}\right) .
$$

Now

$$
\begin{aligned}
f\left(X_{2}\right)= & r_{P}\left(A_{q(1)}^{2}\right) \oplus \ldots \oplus r_{P}\left(A_{q(n)}^{2}\right) \\
& \oplus r_{P}\left(A_{q(n+1)}^{2}\right) \oplus \ldots \oplus r_{P}\left(A_{q(n+l)}^{2}\right) \oplus \alpha \odot r_{Q}\left(B_{2}\right)
\end{aligned}
$$

(where $B_{1}=\{q(1), \ldots, q(n)\}, B_{2}=\{q(1), \ldots, q(n+l)\}$ ). Hence

$$
\begin{aligned}
f\left(X_{2}\right) & <r_{P}\left(A_{q(1)}^{1}\right) \oplus \ldots \oplus r_{P}\left(A_{q(n)}^{1}\right) \oplus \overbrace{\alpha \oplus \ldots \oplus \alpha}^{l \text { times }} \oplus \alpha \odot r_{Q}\left(B_{2}\right) \\
& =r_{P}\left(A_{q(1)}^{1}\right) \oplus \ldots \oplus r_{P}\left(A_{q(n)}^{1}\right) \oplus \alpha \odot\left(r_{Q}\left(B_{2}\right)+l\right) \leq f\left(X_{1}\right) .
\end{aligned}
$$

This ends the proof of Claim 1. 
Applying the claim to $X_{1}=\emptyset$ we get $f(X)<\alpha \odot \beta$ for any non-empty antichain $X$ in $R$ (where $\alpha=r_{P}(P)$ and $\beta=r_{Q}(Q)$ ).

To prove the equality of $f$ and $r_{R}$, we assume (towards a contradiction) that for some antichain $X$, with a minimal $f$-value,

$$
r_{R}(X)=\gamma<f(X) .
$$

The idea is to extend the antichain $X$ to some antichain $X^{\prime}$ that satisfies a corresponding formula, contrary to the minimality of $f(X)$.

We represent $X$ as $\bigcup\left\{A_{q} \mid q \in B\right\}$, where $B=\{q(i) \mid i=1, \ldots, n\}$, and write

$$
f(X)=r_{P}\left(A_{q(1)}\right) \oplus \ldots \oplus r_{P}\left(A_{q(n)}\right) \oplus \alpha \odot r_{Q}(B) .
$$

Then $\gamma<f(X)$ implies, by (1), that

$$
\gamma=\alpha_{1} \oplus \ldots \oplus \alpha_{n} \oplus \ldots \oplus \alpha_{n+l} \oplus \alpha \odot \beta_{0}
$$

where $l \in \omega, \alpha_{i} \leq r_{P}\left(A_{q(i)}\right)$ and $\beta_{0}+l \leq r_{Q}(B)$ by Lemma 1.8.

We shall define $X^{\prime} \supset X$ with $f\left(X^{\prime}\right) \geq \gamma$. Thus $r_{R}\left(X^{\prime}\right)<\gamma \leq f\left(X^{\prime}\right)$ contradicts the minimality of $f(X)$. First a simple observation.

CLAIM 2. If $B$ is an antichain in $Q$ such that $\operatorname{rk}_{\mathcal{A}}(B)=\beta$ and $\beta_{0}<\beta$ is an ordinal such that $\beta_{0}+l \leq \beta$ (where $\left.l \in \omega\right)$, then there is an antichain $B^{\prime} \supset B$ with $\operatorname{rk}_{\mathcal{A}}\left(B^{\prime}\right) \geq \beta_{0}$ and such that $B^{\prime} \backslash B$ contains $l$ points.

Proof. Define inductively $B \subset B_{1} \subset \ldots \subset B_{l}$ by $\operatorname{rk}_{\mathcal{A}}\left(B_{1}\right)=\beta_{0}+$ $(l-1), \ldots, \mathrm{rk}_{\mathcal{A}}\left(B_{l}\right)=\beta_{0}$. Then $B_{l} \backslash B$ contains at least $l$ points, and any $B \subset B^{\prime} \subseteq B_{l}$ containing exactly $l$ additional points concludes the claim.

The construction of $X^{\prime}$ is done in two steps. Since $\beta_{0}+l \leq r_{Q}(B)$, by Claim 2, we first add $l$ new points to $B$ and obtain an antichain $B^{\prime} \supset$ $B$ in $Q$ with $r_{Q}\left(B^{\prime}\right) \geq \beta_{0}$. Having defined $B^{\prime}$, pick for each $q \in B^{\prime}$ an antichain $A_{q}^{\prime}$ in $P$ that extends $A_{q}$ (if $q \in B$ ) and with rank as given by the corresponding ordinal $\alpha_{i}$. Then $f\left(X^{\prime}\right) \geq \gamma$ follows. (To argue that $X^{\prime} \neq X$ consider separately the case $l=0$, in which $\alpha_{i}<r_{P}\left(A_{q(i)}\right)$ for some $i$, and the case $l>0$.)

2. The generalized Hausdorff hierarchy. Fix an ordinal $\varrho \geq 1$ which will denote antichain ranks. We shall define and study in this section the class $\mathcal{H}^{\varrho}$ which is the least class of posets that contain all the well-founded posets with antichain rank $\leq \varrho$ and is closed under inversion and lexicographic sums. We shall stratify $\mathcal{H}^{\varrho}$ in the form $\mathcal{H}^{\varrho}=\bigcup_{\alpha \in \text { Ord }} \mathcal{H}_{\alpha}^{\varrho}$ and investigate the properties of $\mathcal{H}_{\alpha}^{\varrho}$. In particular we prove here that for any $P \in \mathcal{H}_{\alpha}^{\varrho}$, $\operatorname{rk}_{\mathcal{A}}(P) \leq \varrho^{\alpha}$ (Hessenberg based exponentiation). 
Definition 2.1. Let $\varrho \geq 1$ be our fixed ordinal. By induction on $\alpha \in$ Ord define the class $\mathcal{H}_{\alpha}^{\varrho}$ as follows:

1. $\mathcal{H}_{0}^{\varrho}=\{\mathbf{1}\}$ consists of a single poset 1 containing just one element.

2. $\mathcal{H}_{1}^{\varrho}$ is the class of all posets $P$ with $\operatorname{rk}_{\mathcal{A}}(P) \leq \varrho$ and such that $P$ or $P^{*}$ (the inverse of $P$ ) is well-founded. So $\mathcal{H}_{1}^{1}$ is the class of all well-ordered sets and their inverses (i.e., order types of ordinals and inverses of ordinals).

3. For any limit ordinal $\delta, \mathcal{H}_{\delta}^{\varrho}=\bigcup_{\alpha<\delta} \mathcal{H}_{\alpha}^{\varrho}$.

4. If $\beta=\alpha+1$ is a successor ordinal, then $\mathcal{H}_{\beta}^{\varrho}$ is the class of all posets $P$ that are a lexicographic sum of the form

$$
P=\sum_{i \in I} P_{i}
$$

where $P_{i} \in \mathcal{H}_{\alpha}^{\varrho}$ and $I$ is a poset with $\operatorname{rk}_{\mathcal{A}}(I) \leq \varrho$ and such that $I$ or $I^{*}$ is well-founded, that is, $I$ is in $\mathcal{H}_{1}^{\varrho}$.

Finally, set $\mathcal{H}^{\varrho}=\bigcup_{\alpha \in \text { Ord }} \mathcal{H}_{\alpha}^{\varrho}$ and $\mathcal{H}=\bigcup_{1 \leq \varrho \in \text { Ord }} \mathcal{H}^{\varrho}$.

We first state some simple properties concerning this hierarchy of posets $\mathcal{H}^{\varrho}$.

LEMma 2.2. 1. $\mathcal{H}^{\varrho}$ is the least class that contains the well-founded posets of antichain ranks $\leq \varrho$ and is closed under lexicographic sums and inverses. In particular, $\mathcal{H}^{1}$ is the class originally defined by Hausdorff as the least class containing the ordinals that is closed under inverses and sums.

2. Each $\mathcal{H}_{\alpha}^{\varrho}$ and $\mathcal{H}^{\varrho}$ is closed under restrictions.

3. If $P \in \mathcal{H}^{\varrho}$ then $P$ is scattered and satisfies the FAC.

4. $\operatorname{aug}\left(\mathcal{H}^{\varrho}\right)$ (the collection of all augmentations of posets in $\left.\mathcal{H}^{\varrho}\right)$ is closed under lexicographic sums, inverses, restrictions, and (naturally) augmentations. Every poset in $\operatorname{aug}\left(\mathcal{H}^{\varrho}\right)$ is scattered.

Proof. For item 1, we shall only indicate the proof that $\mathcal{H}^{\varrho}$ is closed under lexicographic sums. We prove by induction on $\alpha$ that if $I \in \mathcal{H}_{\alpha}^{\varrho}$ and $P_{i} \in \mathcal{H}^{\varrho}$ for each $i \in I$, then $\sum_{i \in I} P_{i} \in \mathcal{H}^{\varrho}$. The case $\alpha=1$ is obvious by the definition of the hierarchy, and the case of $\alpha$ a limit ordinal is trivial. Let $I \in \mathcal{H}_{\alpha+1}^{\varrho}$ and for each $i \in I$, let $P_{i} \in \mathcal{H}^{\varrho}$ be given. We have $I=\sum_{j \in J} L_{j}$ with $L_{j} \in \mathcal{H}_{\alpha}^{\varrho}$, and $J \in \mathcal{H}_{1}^{\varrho}$. Then $P=\sum_{i \in I} P_{i}=\sum_{j \in J}\left(\sum_{i \in L_{j}} P_{i}\right)$. Now because $J \in \mathcal{H}_{1}^{\varrho}$, we have $P \in \mathcal{H}^{\varrho}$. Items 2,3 and 4 are obvious (note that the last statement of item 4 follows from Lemma 1.2).

TheOREM 2.3. If $R \in \mathcal{H}_{\alpha}^{\varrho}$ then $\operatorname{rk}_{\mathcal{A}}(R) \leq \varrho^{\mathrm{H} \alpha}$ (the superscript $\mathrm{H}$ refers to the Hessenberg based exponentiation, and we omit it below).

Proof. The proof is by induction on $\alpha$. If $\alpha=0$ and $R \in \mathcal{H}_{0}^{\varrho}$, then $R$ consists of a single point and so $\operatorname{rk}_{\mathcal{A}}(R)=1$. The case $\alpha=1$ follows from the definition: if $R \in \mathcal{H}_{1}^{\varrho}$ then $\operatorname{rk}_{\mathcal{A}}(R) \leq \varrho=\varrho^{1}$. If $\alpha$ is a limit ordinal the 
inductive claim follows trivially, and so we assume that $R \in \mathcal{H}_{\alpha+1}^{\varrho}$ and the lemma holds for $\mathcal{H}_{\alpha}^{\varrho}$.

So $R=\sum_{i \in I} P_{i}$ where $P_{i} \in \mathcal{H}_{\alpha}^{\varrho}$ and $I \in \mathcal{H}_{1}^{\varrho}$. It follows from the inductive assumption that $\operatorname{rk}_{\mathcal{A}}\left(P_{i}\right) \leq \varrho^{\alpha}$, and it is not difficult to find a single poset $P$ with $\operatorname{rk}_{\mathcal{A}}(P) \leq \varrho^{\alpha}$ and such that $P_{i} \subseteq P$ for each $i \in I$ (just align the $P_{i}$ 's along some ordinal). Then $R \subseteq P \cdot I$ and hence $\operatorname{rk}_{\mathcal{A}}(R) \leq \operatorname{rk}_{\mathcal{A}}(P \cdot I)=$ $\operatorname{rk}_{\mathcal{A}}(P) \odot \operatorname{rk}_{\mathcal{A}}(I) \leq \varrho^{\alpha} \odot \varrho=\varrho^{\alpha+1}$. (Use Lemma 1.11.)

3. A generalization of Hausdorff's theorem. This is the main section of our paper.

A quite useful and well-known observation is that every poset $P$ has a cofinal well-founded set. (Recall that $H \subseteq P$ is cofinal iff every $p \in P$ has an extension in $H$.) To prove this, define $h_{\xi}$ by induction until $\left\{h_{\xi} \mid \xi<\alpha\right\}=$ $H_{\alpha}$ is cofinal. The inductive requirement is that for every $\xi_{1}<\xi_{2}$ we have $h_{\xi_{2}} \nless^{P} h_{\xi_{1}}$; this ensures that $H_{\alpha}$ is well-founded.

Given a cofinal well-founded sequence $H=\left\{h_{\xi} \mid \xi<\alpha\right\} \subseteq P$ as above, we define the stratification of $P$ (induced by $H$ ) as the sequence of disjoint subposets

$$
A\left(h_{\xi}\right)=\left\{p \in P \mid p \leq^{P} h_{\xi} \text { but } p \not^{P} h_{\xi^{\prime}} \text { for } \xi^{\prime}<\xi\right\}, \quad \text { for } h_{\xi} \in H .
$$

We consider $A\left(h_{\xi}\right)$ as a restriction of $P$.

Suppose that $P$ satisfies FAC and $H^{\prime}$ is a well-founded subset of $P$. We define

$$
\left(\leq H^{\prime}\right)=\bigcup_{h \in H^{\prime}}(\leq h) \text { and } F\left(H^{\prime}\right)=P \backslash\left(\leq H^{\prime}\right)
$$

We say that $p \in F\left(H^{\prime}\right)$ is minimal over $H^{\prime}$ if for every $q \in F\left(H^{\prime}\right) \cap(\leq p)$,

$$
h \leq q \text { if and only if } h \leq p \text { for every } h \in H .
$$

If $P$ satisfies the FAC, then the cofinal well-founded sequence $H$ can be defined in such a way that $P$ is an augmentation of the lexicographic sum $\sum_{h \in H} A(h)$ obtained from the stratification of $P$ induced by $H$ (using the existence of minimal point). Towards this aim we bring the following lemma.

LEMMA 3.1. If $P$ is a FAC poset, then there exists a cofinal well-founded sequence $H=\left\{h_{\xi} \mid \xi<\alpha\right\}$ such that, for every $\xi<\alpha, h_{\xi}$ is minimal over $H_{\xi}=\left\{h_{\mu} \mid \mu<\xi\right\}$.

Proof. The construction of $H$ is done inductively as before, but with the following change. Suppose that $H_{\xi}=\left\{h_{\mu} \mid \mu<\xi\right\}$ has been defined with each $h_{\mu}$ minimal over its set of predecessors. If $H_{\xi}$ is cofinal in $P$, then we are done. Otherwise $F_{\xi}:=F\left(H_{\xi}\right)$ is non-empty, and we must find some minimal element $h=h_{\xi} \in F_{\xi}$ over $H_{\xi}$. Suppose that no such $h$ can be found, and construct an infinite descending sequence $p(0)>^{P} \ldots>^{P} p(i)>^{P} \ldots$ 
in $F_{\xi}$ and corresponding members $h_{\mu(i)}$ of $H_{\xi}$ such that for each $i<\omega$,

$$
h_{\mu(i)}<{ }^{P} p(i) \text { but } h_{\mu(i)} \nless^{P} p(i+1) .
$$

Indeed, $p(0) \in F_{\xi}$ is arbitrarily chosen, and having defined $p(i)$ we use the supposition that $p(i)$ is not minimal over $H_{\xi}$ to find $p(i+1)<{ }^{P} p(i)$ in $F_{\xi}$ with a different type: for some $h_{\mu(i)}$ in $H_{\xi}$, (9) holds. Since $P$ satisfies the FAC and $H_{\xi}$ is well-founded, $H_{\xi}$ is a well-founded FAC poset, and hence there must be $i<j$ with $h_{\mu(i)}<^{P} h_{\mu(j)}$ (apply Lemma 1.1). But then

$$
h_{\mu(i)}<{ }^{P} h_{\mu(j)}<{ }^{P} p(j) \leq{ }^{P} p(i+1)
$$

contradicts (9).

Now we look at the stratification $\langle A(h) \mid h \in H\rangle$ obtained by a wellfounded cofinal sequence $H$ with the "minimality condition" as in the lemma. Form the lexicographic sum $S=\sum_{h \in H} A(h)$ where $A(h)$, as defined in (8), is the collection of points $\leq^{P} h$ that are not in any previous $A\left(h^{\prime}\right)$. Then the universe of $S$ is the universe of $P$, since $H$ is cofinal. We claim that $P$ augments $S$. Indeed, assume that $p_{1}<^{S} p_{2}$. There are two possibilities:

1. For some $h \in H, p_{1}, p_{2} \in A(h)$. Then $p_{1}<^{S} p_{2}$ iff $p_{1}<^{P} p_{2}$ by definition.

2. $p_{1} \in A\left(h^{1}\right)$ and $p_{2} \in A\left(h^{2}\right)$, and $p_{1}<^{S} p_{2}$ because $h^{1}<^{P} h^{2}$. Then by the "special minimality property" any point in $A\left(h^{2}\right)$ is above $h^{1}$ and thus any point in $A\left(h^{1}\right)$ is $<^{P}$-below any point in $A\left(h^{2}\right)$.

Corollary 3.2. Suppose $P$ is a FAC poset with $\operatorname{rk}_{\mathcal{A}}(P) \leq \varrho$. If for every $x \in P,(\leq x) \in \operatorname{aug}\left(\mathcal{H}^{\varrho}\right)$, then $P \in \operatorname{aug}\left(\mathcal{H}^{\varrho}\right)$.

Proof. Find first an ordinal $\alpha$ such that $(\leq x) \in \operatorname{aug}\left(H_{\alpha}^{\varrho}\right)$ for every $x \in P$, and we will prove that $P \in \operatorname{aug}\left(H_{\alpha+1}^{\varrho}\right)$. Construct a cofinal wellfounded sequence $H \subseteq P$ as in Lemma 3.1, and form the lexicographic sum

$$
S=\sum_{h \in H} A(h)
$$

of the resulting stratification. We consider the following facts:

1. $P$ augments $S$.

2. Each $A(h)$ is a restriction of $(\leq h)$ to some subset, and hence $A(h) \in$ $\operatorname{aug}\left(\mathcal{H}_{\alpha}^{\varrho}\right)$ (as $\mathcal{H}_{\alpha}^{\varrho}$ and hence $\operatorname{aug}\left(\mathcal{H}_{\alpha}^{\varrho}\right)$ are closed under restrictions). Say $A(h)$ augments $A^{0}(h) \in \mathcal{H}_{\alpha}^{\varrho}$.

3. $H$ is well-founded and $\operatorname{rk}_{\mathcal{A}}(H) \leq \varrho$ (as $H \subseteq P$ ). Thus $H \in \mathcal{H}_{1}^{\varrho}$.

4. So $S^{0}=\sum_{h \in H} A^{0}(h) \in \mathcal{H}_{\alpha+1}^{\varrho}$. Now, $S$ augments $S^{0}$ since each $A(h)$ augments $A^{0}(h)$.

5. But $S$ augments $S^{0}$ and $P$ augments $S$ imply that $P$ augments $S^{0}$. Thus $P \in \operatorname{aug}\left(\mathcal{H}_{\alpha+1}^{\varrho}\right)$.

This proves Corollary 3.2. 
Since $\mathcal{H}_{1}^{\varrho}$ also contains all inverses of well-founded posets of antichain rank $\leq \varrho$, the same result holds for every poset $P$ with $\operatorname{rk}_{\mathcal{A}}(P) \leq \varrho$ and such that each $(\geq x)$ is in $\operatorname{aug}\left(\mathcal{H}^{\varrho}\right)$.

Corollary 3.3. 1. If $P$ is a FAC poset with $\operatorname{rk}_{\mathcal{A}}(P) \leq \varrho$, but $P \notin$ $\operatorname{aug}\left(\mathcal{H}^{\varrho}\right)$, then for some $p \in P,(\leq p) \notin \operatorname{aug}\left(\mathcal{H}^{\varrho}\right)$ and $(\geq p) \notin \operatorname{aug}\left(\mathcal{H}^{\varrho}\right)$.

2. If $P$ is a FAC poset with $\operatorname{rk}_{\mathcal{A}}(P) \leq \varrho$ but $P \notin \operatorname{aug}\left(\mathcal{H}^{\varrho}\right)$, then $\mathbb{Q}$ is embeddable in $P$, that is, $P$ is not scattered.

Pr o o f. We first prove 1. Suppose that $P$ is a FAC poset with $\operatorname{rk}_{\mathcal{A}}(P) \leq$ $\varrho$, and let $P^{0}=\left\{p \in P \mid(\leq p) \in \operatorname{aug}\left(\mathcal{H}^{\varrho}\right)\right\}$ and $P^{1}=\{p \in P \mid(\geq p) \in$ $\left.\operatorname{aug}\left(\mathcal{H}^{\varrho}\right)\right\}$. Assume that $P=P^{0} \cup P^{1}$. We must prove that $P \in \operatorname{aug}\left(\mathcal{H}^{\varrho}\right)$. $P^{0}$ is an initial segment of $P$, and $P^{1}$ a final segment (that is, $(\geq q) \subseteq P^{1}$ for every $\left.q \in P^{1}\right)$. By Corollary 3.2 , both $P^{0}$ and $P^{1}$ are in $\operatorname{aug}\left(\mathcal{H}^{\varrho}\right)$ : Say $Q^{0}, Q^{1} \in \mathcal{H}^{\varrho}$ are such that $P^{i} \in \operatorname{aug}\left(Q^{i}\right)$ for $i=0,1$. There are two cases: either for every $x \in P^{0}$ and $y \in P^{1}$ we have $x \leq^{P} y$, or else some $x \in P^{0}$ and $y \in P^{1}$ are incomparable.

In the first case $P$ is the sum $P^{0}+\left(P^{1} \backslash P^{0}\right)$ of two posets that are in $\operatorname{aug}\left(\mathcal{H}^{\varrho}\right)$, and hence $P$ is in $\operatorname{aug}\left(\mathcal{H}^{\varrho}\right)$ as well (because $\mathcal{H}^{\varrho}$ is closed under sums).

In the second case there are two incomparable points in $P$ and thus $\varrho>1$. Hence $\mathcal{H}_{1}^{\varrho}$ contains the poset consisting of two incomparable members, and so $\mathcal{H}^{\varrho}$ is closed under perpendicular sums. As $P$ is an augmentation of $Q^{0} \perp\left(Q^{1} \backslash Q^{0}\right)$ it follows that $P \in \operatorname{aug}\left(\mathcal{H}^{\varrho}\right)$.

The second part of our lemma requires that we find an embedding $f$ of the rational chain $\mathbb{Q}$ into $P$, in the case where $P$ is a FAC poset with antichain rank $\leq \varrho$ and such that $P \notin \operatorname{aug}\left(\mathcal{H}^{\varrho}\right)$.

Fix an enumeration $q_{n}$ of the rational numbers, and decide in the $n$th step of the construction the value of $f\left(q_{n}\right) \in P$. The inductive assumption is that, for distinct $i, j<n$, if $q_{i}<^{\mathbb{Q}} q_{j}$ then $f\left(q_{i}\right)<^{P} f\left(q_{j}\right)$ and the $P$-interval

$$
\left[f\left(q_{i}\right), f\left(q_{j}\right)\right]=\left\{p \in P \mid f\left(q_{i}\right) \leq p \leq q_{j}\right\}
$$

is not in $\operatorname{aug}\left(\mathcal{H}^{\varrho}\right)$, and similarly that $\left(\leq f\left(q_{i}\right)\right)$ and $\left(\geq f\left(q_{i}\right)\right)$ are not in $\operatorname{aug}\left(\mathcal{H}^{\varrho}\right)$. The first part of the lemma is used at each step. Thus we construct $f$ which is an embedding of $\mathbb{Q}$ into $P$.

Since the second part of the corollary is our main result we restate it for conclusion:

THEOREM 3.4. $\operatorname{aug}\left(\mathcal{H}^{\varrho}\right)$, which is the closure of the class of all wellfounded posets with antichain rank $\leq \varrho$ under inversion, lexicographic sums, and augmentation, contains the class of all scattered FAC posets with ranks $\leq \varrho$. So $\operatorname{aug}(\mathcal{H})$, which is the closure of the well-founded posets with FAC 
under inversion, lexicographic sums, and augmentation, is the class of all scattered FAC posets.

Hausdorff's theorem is obtained for $\varrho=1$.

4. On the need for augmentations. Is it possible to prove a stronger statement in which there is no need for augmentations? That is, is it true that $\mathcal{H}$ already contains all scattered FAC posets? We shall show in this section that this is not the case. We first make the following

Definition. Let $P$ be a poset. A subset $S$ of $P$ is called a strong interval of $P$ iff for every $x, y \in S$ and $p \in P \backslash S$, we have $p \leq x$ iff $p \leq y$, and $p \geq x$ iff $p \geq y$.

In other words, $S$ is a strong interval if any $p \in P \backslash S$ is either greater than all members of $S$, or smaller, or incomparable with all of them. For instance, $\emptyset, P$, and every singleton are strong intervals of any poset $P$. Also, if $P$ is a lexicographic sum $\sum_{q \in Q} P_{q}$, then each $P_{q}$ is a strong interval of $P$.

The following trivial observations are useful: (1) Any strong interval is an interval (a convex set). (2) If $S$ is a strong interval of $P$, if $s, s^{\prime} \in S$ and $p \in P$ is such that $p$ is comparable with $s$ but not with $s^{\prime}$, then $p \in S$.

We describe a FAC poset $P$ that is not in $\mathcal{H}$. Let $A=\left\{a_{n} \mid n \in \omega\right\}$ and $B=\left\{b_{n} \mid n \in \omega\right\}$ be disjoint countable sets, and let $Q=A \cup B$. Let $<$ be the ordering on $Q$ defined by

$$
a_{0}<a_{1}<a_{2}<a_{3}<\ldots<b_{3}<b_{2}<b_{1}<b_{0} .
$$

Let $\{0,1\}$ be the linear ordering of two points $0<1$, and then let

$$
P=Q \times\{0,1\}
$$

be the Cartesian product. Then $P$ has a least element $p^{-}=\left\langle a_{0}, 0\right\rangle$ and a greatest element $p^{+}=\left\langle b_{0}, 0\right\rangle$.

LEMma 4.1. The strong intervals of $P$ are $\emptyset$, the singletons, and the sets of the form $P \backslash X$ where $X \subseteq\left\{p^{-}, p^{+}\right\}$. Hence every strong interval of $P$ that contains at least two points contains a restriction isomorphic to $P$.

P r o of. Clearly, each of these sets, except the singletons and the empty set, is a strong interval of $P$, and contains a restriction isomorphic to $P$. For example, $S=P \backslash\left\{p^{+}\right\}$is a strong interval because $p^{+}$is greater than all members of $S$. Also, $S \backslash\left\{p^{-}\right\}$contains a restriction isomorphic to $P$.

Now let $S$ be any strong interval of $P$ containing at least two points; we shall prove that $S$ has the required form. Let $Q_{0}=Q \times\{0\}$ and $Q_{1}=Q \times\{1\}$.

We claim that it is not possible for $S$ to be contained in $Q_{0}$. Indeed, in such a case pick two points in $S$ : they are of the form $\langle i, 0\rangle,\langle j, 0\rangle$ with $i<^{Q} j$. But then $e=\langle i, 1\rangle \notin S$ contradicts the assumption that $S$ is a strong interval, because $e$ is above $\langle i, 0\rangle$ but is incomparable with $\langle j, 0\rangle$. In a 
similar vein $S$ cannot be contained in $Q_{1}$. Thus we conclude that $S$ contains a point $\langle i, 0\rangle$ from $Q_{0}$ and a point $\langle j, 1\rangle$ from $Q_{1}$. The proof now proceeds in four cases.

Case 1: Both $p^{-}$and $p^{+}$are in $S$. Then $S=P$ because $S$ is convex.

CASE $2: p^{+} \notin S, p^{-} \in S$. Since $p^{+} \notin S$, but $\langle j, 1\rangle \in S$ by our conclusion, necessarily $j \neq b_{0}$ and then $\left\langle b_{0}, 0\right\rangle$ is incomparable with $\langle j, 1\rangle$. Yet it is above $p^{-}$, and thus $\left\langle b_{0}, 0\right\rangle \in S$. This is because $p^{-} \in S$ and $S$ is an interval $Q_{0} \subset S$. A similar argument can show that $Q_{1} \backslash\left\{p^{+}\right\} \subset S$ : this follows because any $\langle k, 1\rangle$ is incomparable with $\left\langle b_{0}, 0\right\rangle \in S$ but is above $p^{-}$. Thus $S=P \backslash\left\{p^{+}\right\}$, and in particular $S$ contains an isomorphic copy of $P$.

CASE 3: $p^{+} \in S, p^{-} \notin S$. This case is symmetric to Case 2 , and $S=$ $P \backslash\left\{p^{-}\right\}$again contains a restriction isomorphic to $P$.

CASE $4: p^{-}, p^{+} \notin S$. Then $\left\langle b_{0}, 0\right\rangle$ is in $S$ as it is incomparable with $\langle j, 1\rangle$ but comparable with $\langle i, 0\rangle$. Similarly, $\left\langle a_{0}, 1\right\rangle \in S$. Except for $p^{-}$, any pair in $Q_{0}$ is incomparable with $\left\langle a_{0}, 1\right\rangle$ and comparable with $\left\langle b_{0}, 0\right\rangle$, and thus $Q_{0} \backslash\left\{p^{-}\right\} \subset S$. Similarly, $Q_{1} \backslash\left\{p^{+}\right\} \subset S$. Thus $S=P \backslash\left\{p^{-}, p^{+}\right\}$, and again $S$ contains an isomorphic copy of $P$.

Corollary 4.2. $P \notin \mathcal{H}$.

Proof. Look at

$$
\mathcal{H}^{\prime}=\{Q \in \mathcal{H} \mid \text { no restriction of } Q \text { is isomorphic to } P\} .
$$

Prove that $\mathcal{H}^{\prime}$ contains all FAC well-founded posets and their inverses, and that it is closed under restrictions, inverses, and lexicographic sums. (Hence $\mathcal{H}^{\prime}=\mathcal{H}$ and so $P \notin \mathcal{H}$.)

Indeed, the main point is the proof that if $P$ can be represented as a lexicographic sum $R=\sum_{q \in Q} P_{q}$, then either a restriction of $Q$ is isomorphic to $P$, or else a restriction of some $P_{q}$ is isomorphic to $P$. To prove this, consider first the case where any $P_{q}$ is a singleton or the empty poset. Then $Q$ is isomorphic to $P$. Otherwise some $P_{q}$ contains two points. But each $P_{q}$ is a strong interval of $R$, and hence this $P_{q}$ contains a restriction isomorphic to $P$.

\section{References}

[1] U. Abraham, A note on Dilworth's theorem in the infinite case, Order 4 (1987), $107-125$.

[2] R. Bonnet et M. Pouzet, Extension et stratification d'ensembles dispersés, C. R. Acad. Sci. Paris Sér. A 168 (1969), 1512-1515.

[3] P. W. Carruth, Arithmetic of ordinals with applications to the theory of ordered abelian groups, Bull. Amer. Math. Soc. 48 (1942), 262-271. 
[4] R. Fraïssé, Theory of Relations, Stud. Logic Found. Math. 118, North-Holland, 1986.

[5] F. Hausdorff, Grundzüge einer Theorie der geordneten Mengen, Math. Ann. 65 (1908), 435-505.

[6] G. Hessenberg, Grundbegriffe der Mengenlehre, Abh. Friesschen Schule neue Folge 4 (1906).

[7] A. Lévy, Basic Set Theory, Springer, 1979.

[8] W. Sierpiński, Cardinal and Ordinal Numbers, Monograf. Mat. 34, PWN, Warszawa, 1958.

Department of Mathematics and Computer Science

Ben-Gurion University

Be'er Sheva, Israel

E-mail: abraham@cs.bgu.ac.il
Laboratoire de Mathématiques Université de Savoie 73011 Chambéry, France E-mail: bonnet@in2p3.fr 